\title{
THE ROLES OF EFFICIENCY AND COMPLEXITY IN THE PROCESSING OF VERB PARTICLE CONSTRUCTIONS
}

\author{
GONNERMAN, Laura* \\ McGill University
}

\begin{abstract}
Recent theories have proposed that processing difficulty affects both individuals' choice of grammatical structures and the distribution of these structures across languages of the world (Hawkins, 2004). Researchers have proposed that performance constraints, such as efficiency, integration, and storage costs, drive languages to choose word orders that minimize processing demands for individual speakers (Hawkins, 1994; Gibson, 2000). This study investigates how three performance factors, adjacency, dependency, and complexity, affect reading times for sentences with verb-particle constructions. Results from a self-paced reading task indicate that reading times increase with each performance factor, such that shifted sentences, more dependent verb-particle constructions, and more complex noun phrases are more difficult. More importantly, I explore the relative weightings and interactions of the performance factors. The results support the notion that processing ease affects grammaticalization, such that those structures which are more easily processed by individuals (subject relatives and adjacent dependent constituents) are also more common crosslinguistically (Keenan \& Hawkins, 1987).
\end{abstract}

Keywords: sentence processing; word order; verb particle; linguistic complexity; relative clause. 


\section{Introduction}

Many factors can affect the comprehension of sentences. For example, word order has been shown to play an important role in processing ease (c.f. Stallings, MacDonald, O’Seaghdha, 1998; Hawkins, 1994, 2004), such that sentence (1) is much easier to process than sentence (2):

(1) The boy will throw [the girl $]_{\mathrm{NP}-1}$ [the bright orange ball] .NP-2

(2) The boy will throw [the bright orange ball $]_{\mathrm{NP}}$ [to the girl].PP

All of the components of sentence (1) can be recognized in fewer words than in (2). Hawkins (1994, 2004) has proposed that this early recognition facilitates processing ease.

The semantic relationship between words can also affect ease of comprehension (Lohse, Hawkins, \& Wasow, 2004; Gonnerman and Hayes, 2005). For example, (3) is much easier to process than (4):

(3) The man will chew the food up.

(4) The man will chew the kids out.

In sentence (3), 'chew' depends much less on 'up' for its meaning, whereas 'chew' depends highly on 'out' for proper interpretation of sentence (4). Thus, processing is facilitated when the dependency relationship between words is minimized as in sentence (3).

Another factor that can affect processing ease is the complexity of phrases (Keenan and Hawkins, 1987). Phrase (5), a subject relative clause, is less complex and hence much easier to process than (6), a direct-object relative clause:

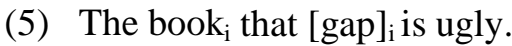

(6) The book $k_{i}$ that John owned [gap $]_{i}$.

Subject relatives require fewer words between the head (book) and relativized gap than direct-object relatives and thus facilitate processing (Hawkins, 2004).

Hawkins' (1994, 2004) provides a unified account for why (1), (3), and (5) are all easier to process than (2), (4), and (6). Hawkins suggests that syntactic and semantic factors, such as word order, dependency between sentence elements, and complexity facilitate processing when they minimize the syntactic or semantic domain of a sentence. Thus, sentence (1) was easier to process since its word order allowed for the earlier recognition of all sentence constituents. Sentence (3) was easier to process since 'chew' is less dependent on 'up' in (3) than 'out' in (4) for its complete meaning; thereby reducing the semantic dependency relationship across the sentence.

The complexity of phrases like (5) and (6), or relative clauses, has been shown to affect processing ease, with subject relatives (5) processed more easily than direct object relatives (6) (Keenan and Hawkins, 1987). Complexity of relative clauses has not only been linked to processing ease, but has also been shown to affect the distribution of relative clauses across languages of the world, with less complex relatives occurring more often (Keenan and Comrie, 1977). Hawkins (2004) has provided an account for 
why this relationship between performance and grammars exists. His account links linguistic efficiency with processing ease in individuals, and suggests that individual performance shapes the grammar of a particular language. The result of which is the grammaticalization of those structures that most facilitate processing. Hawkins suggests that this link between performance and grammars results in the relative prevalence of more easily processed structures across languages of the world (c.f. Keenan and Comrie, 1977; Keenan and Hawkins, 1987).

This paper examines the comparative role of three performance factors (adjacency, semantic dependency, and noun phrase (NP) complexity) on the comprehension of sentences that have variable word orders. I examine not only how these factors interact and their relative impact on performance, but how processing is affected when syntactic and semantic factors work in opposition to one another. The processing outcome of opposing forces can speak to the relative importance of certain factors on comprehension and thus, perhaps, the importance of their grammaticalization across languages of the world.

\subsection{Efficiency and Complexity in Sentence Processing}

Hawkins' (1994, 2004) account of sentence processing efficiency is based on factors that affect the recognition domain of sentence constituents. His theory accounts for ordering preferences and processing ease based on many underlying causes of processing efficiency, some of which include: communication speed, articulatory effort, errors, and ambiguity. Thus, his efficiency approach is broader in scope than other sentence processing theories which rely on more specific processing constraints such as working memory load and prediction-content congruence (cf. Gibson, 2000; Lewis \& Nakayama, 2002).

In Hawkins' account of syntactic processing, he attributes the difficulty of structures, and thus the efficiency of processing, to the distance between dependent items in a sentence, otherwise known as the size of the syntactic domain. Hawkins proposes that minimizing a syntactic domain facilitates processing by reducing linguistic complexity and increasing processing efficiency. Thus, processing is made easier when all of the constituents of a sentence are recognized as early as possible, and the syntactic domain is minimized. Different orderings of constituents generally involve changes in recognition time. Therefore, differences in word order may change processing ease since one order may allow earlier constituent recognition than another.

For example, according to Hawkins, sentence (8) is more easily processed than sentence (7) because sentence (7) requires a longer constituent recognition domain than sentence (8). 
(7) $[\mathrm{I}]_{\mathrm{NP}-1}[\text { lent }]_{\mathrm{VP}}$ [the book about whales in the Atlantic Ocean $]_{\mathrm{NP}-2}$ [to Jim $]_{\mathrm{PP}}$.

$\begin{array}{llllllllllll}1 & 2 & 3 & 4 & 5 & 6 & 7 & 8 & 9 & 10 & 11 & 12\end{array}$

(8) $[\mathrm{I}]_{\mathrm{NP}-1}[\text { lent }]_{\mathrm{VP}}$ [to Jim $]_{\mathrm{PP}}$ [the book about whales in the Atlantic Ocean $]_{\mathrm{NP}-2}$.

$\begin{array}{llllllllllll}1 & 2 & 3 & 4 & 5 & 6 & 7 & 8 & 9 & 10 & 11 & 12\end{array}$

That is, for sentence (7), 11 words must be processed in order to recognize all of the constituents of the sentence: the subject noun phrase (NP-1), verb phrase (VP), direct object NP (NP-2), and prepositional phrase (PP). However, sentence (8) only requires the listener to process 5 words before encountering the final sentence constituent (NP-2). Therefore, sentence (8) should be easier to process due to its shorter recognition domain.

Other theorists have emphasized the roles of both integration and storage costs (Gibson, 2000), as well as expectation, (Lewis and Nakayama, 2002) on processing difficulty. Gibson (2000) suggests that it is not only the number of words between sentence elements that affects processing, but the integration of new words and the storing of constituents across the sentence that affect processing difficulty. His account stresses the importance of working memory load, suggesting that all listeners work with working memory constraints that affect processing difficulty when sentence orderings have heavy storage or integration requirements.

Lewis and Nakayama (2002) have suggested that expectation, rather than working memory load or distance, affects processing. They have suggested that listeners predict what will come next in a sentence based on the content they have already processed. On their account processing is easier when sentence content coincides with previously hypothesized parses and difficulty arises when hypotheses and current sentence content do not match.

\subsubsection{Experimental Studies of Hawkins' Syntactic Processing Theory}

Experimental studies have shown that sentences which minimize the constituent recognition domain do occur more frequently in production and are processed more easily in comprehension, supporting Hawkins’ account (1994, 2004).

Consistent with Hawkins' predictions, results of several production experiments have shown that constituent length affects word order preference. For example, Stallings, MacDonald, and O’Seaghdha (1998) showed that speakers generally preferred word orders in which shorter constituents preceded longer ones, thus minimizing the syntactic domain. They demonstrated that the length of a direct object noun phrase (NP) affects word order choice, with participants strongly preferring 'Mary explained [to Sam $]_{P P}$ [the recently published theory of genetic inheritance $]_{N P}$ ' over 'Mary explained [the recently published theory of genetic inheritance $]_{N P}[\text { to Sam }]_{P P}$. Length effects have also been reported for English 
dative alternation constructions, where speakers are more likely to produce shifted structures if the direct object NP is long (Wasow, 1997).

Constituent length effects have also been demonstrated for the placement of particles in verbparticle constructions in both corpus and experimental comprehension studies. Lohse, Hawkins, \& Wasow (2004) demonstrated that sentences in which the verb and particle occurred adjacently were more common in corpora, especially when the direct object NP was long. In a self-paced reading experiment, Gonnerman \& Hayes (2005) showed that listeners processed sentences faster when the verb and particle occurred together and that sentences were read more slowly when a long direct object NP occurred between a verb and particle.

In addition to studies of NP length effects on word order in English, data from cross-linguistic studies show that processing effects on word order are more broadly applicable. Yamashita and Chang (2001) showed that long NP's are preferred in early rather than sentence final position for Japanese, a right-headed language. They found that when sentences offered ordering options, Japanese speakers chose to produce orders that fronted long object NPs. These results are consistent with the idea that in Japanese, processing effort is minimized by "long before short" orderings, which allow all constituents to be processed in the minimum number of words.

These experimental studies provide evidence that both speakers and listeners benefit from the minimization of syntactic domains for several constituent types, different basic word orders, and different languages. These results support Hawkins' theory that constituent length and order influences complexity of sentence structure and thus processing efficiency.

\subsection{The Relationship between Performance and Grammars}

Hawkins (1994, 2004) account goes beyond the linguistic explanation of efficiency to propose that performance factors, like those that underlie processing efficiency and linguistic complexity, are related to the grammaticalized structures within a language. Thus, less complex structures and sentence orderings tend to be grammaticalized more often in languages of the world since they increase processing efficiency in individuals. Therefore, it is likely that orderings that maximize processing efficiency and minimize domains, as in (9), occur more often in languages of the world than those that do not, as in (10). In addition, when word orders are fixed in a language, the ordering of sentence (9) would be the grammaticalized one.

(9) She sent [the doctor $]_{\mathrm{NP}}$ [the box of medical supplies $]_{\mathrm{NP}}$.

$$
\begin{array}{lllll}
1 & 2 & 3 & 4 & 5
\end{array}
$$

(10) She sent $[\text { the box of medical supplies }]_{\mathrm{NP}}[\text { to the doctor }]_{\mathrm{PP}}$.

$\begin{array}{llllllll}1 & 2 & 3 & 4 & 5 & 6 & 7 & 8\end{array}$


Other linguists have long observed this correlation between performance in individual language users and the structure of grammars across languages of the world, suggesting that those structures which are more preferred are also most common across languages. For example, this relationship between performance and grammars, in the case of relative clauses, was described by Keenan and Comrie (1977). Their Accessibility Hierarchy illustrates the relationship between the linguistic complexity of different types of relative clauses and their prevalence across languages of the world.

The entire hierarchy can be described as in (11) below where complexity increases from left to right and prevalence across languages decreases with increasing complexity.

(11) Subject $>$ Direct Object $>$ Indirect Object $>$ Oblique $>$ Genitive

Keenan and Comrie showed that a language which permits the use of a more complex relative must also permit the usage of all relatives of lesser complexity. Therefore, a grammar that has a strategy for relativizing genitives (which are the most complex linguistically) must also have a strategy for relativizing all other relative clauses, as in Japanese. In contrast, Arabic only allows subject relatives (least complex), thus no other relativizations are possible.

A decade later, Keenan and Hawkins (1987) conducted an experiment designed to test whether structures considered linguistically more complex were actually more difficult for individuals to process. In a repetition experiment, they showed that repetition accuracy of relative clauses correlated with their position on the Accessibility Hierarchy, with subject relatives repeated more accurately than object relatives or other relatives, which are farther down the hierarchy. These data are consistent with the notion that performance and grammars are correlated, such that those structures which are more difficult to process by individuals are less prevalent across languages of the world (c.f. Hawkins 1994, 2004).

Hawkins (2004) explains the processing difficulty of individual sentence constituents, such as those reflected in the hierarchy of relative clauses described by Keenan and Comrie (1977) in terms of minimizing domains. Relative clauses consist of a head and a restricting clause which limits the potential referents to which the head can refer (Comrie, 1981). For example, the restricting clause, 'that had a red collar,' when applied to the head, 'the dog,' delimits the number of possible dogs referred to in the sentence 'the dog that had a red collar ran away.' In addition, relative clauses include a gap which acts as a place holder for the head noun in the restricting phrase. For example, in the direct object relative clause (12):

(12) the $\operatorname{man}_{\mathrm{i}}$ that the child saw $[\mathrm{gap}]_{\mathrm{i}}$

the head noun (the man) is the relativized direct object in the implied phrase; the child saw (the man). However, in English relative clauses, the implied direct object need not be overt and instead can be left out and indexed by a gap. 
According to Hawkins (2004), the complexity of a relative clause coincides with the distance between the head noun and the gap of the clause, that is, the minimization of the dependency relations within the clause. Therefore, subject relatives require the shortest distance (smallest domain) between the relative clause head and the gap and are hence the least complex of the relative clauses. For example, the subject relative clause (13):

(13) the teacher ${ }_{\mathrm{i}}$ that $[\text { gap }]_{\mathrm{i}}$ graded the tests

only requires one word, that, between the clause head (teacher) and the gap. However, for a direct object relative clause (14):

(14) the teacher ${ }_{i}$ that the principal called [gap $]_{\mathrm{i}}$

four words intercede between the clause head and gap, resulting in a larger domain and greater complexity.

This distance between head and gap corresponds with the Accessibility Hierarchy of Keenan and Comrie (1977), with the distance between head and gap increasing up the hierarchy, such that with the shortest head to gap distance occurs for subject relatives and the longest for genitive relatives. Thus, Hawkins (2004) provides a mechanistic explanation for the findings of Keenan and Hawkins (1987), suggesting that a shorter distance between head and gap facilitates processing of relative clauses. Hawkins' account also suggests a link between domain minimization, processing ease, and prevalence of relative clauses across languages of the world such that, relative clauses that have shorter recognition domains are easier to process and are also more common across languages of the world (Keenan \& Comrie, 1977).

\subsection{Semantic Relationships in Verb-Particle Constructions}

In addition to syntactic domains, semantic dependencies between words can influence preferred word order and processing performance in language users (Lohse et al., 2004, Gonnerman and Hayes, 2005). In English, verb-particle constructions allow for the examination of both syntactic and semantic domains since they include a verb (e.g., look) and a particle (e.g., up) that can either be produced adjacently as in 'he looked up the word' or separately (with an intervening noun phrase) as in 'he looked the word up'. In addition, the verb and particle have a semantic dependency relationship that varies in the extent to which a verb relies on its particle for the meaning of the complete construction. For example, finish does not rely on up for its interpretation in finish up, whereas chew depends strongly on the particle, out, in chew out for its semantic interpretation. The placement of particles in sentences with verb-particle constructions has long been a subject of interest in the linguistic literature, with researchers describing several phonological, syntactic, semantic, pragmatic, and discourse factors that affect particle placement (e.g., Bolinger, 1971; Gries, 1999, 2002; Live, 1965; van Dongern, 1919, see also articles in Dehé, Jackendoff, McIntryre, \& 
Urban. 2002, for a variety of methodological and theoretical approaches to understanding verb particles in English and other languages).

Lohse et al. (2004) argue that the various factors that affect particle placement can be explained by a processing efficiency approach. Their account of particle placement expands on the work of Hawkins (2004) to include structures that not only minimize syntactic domains, but also semantic ones. In a corpus study, they examined two factors that affect particle placement, namely length of the direct object NP (a syntactic factor) and dependency of the verb and particle (a semantic factor). They showed that as the number of words in the direct object NP increased, the number of split orderings diminished. Thus, the particle was more likely to be placed adjacent to the verb if the direct object NP was long. More importantly, they showed that dependent particles were more likely to be placed adjacent to the verb, thereby minimizing the semantic domain. For example, the sentence 'The teacher will chew out the students' is more common than 'The teacher will chew the students out,' since the former minimizes the semantic domain between a very dependent verb and particle. In contrast, when the verb and particle do not have a high semantic dependency relationship, the verb and particle adjacency is not more likely than non-adjacency. For, example, the sentence 'The children will finish up the meal' is just as common as 'The children will finish their meal up.'

The psychological validity of this notion of dependency has been examined by Gonnerman and Hayes (2005). They found that participants were sensitive to the dependency relationship between verbs and particles based on judgments of the semantic similarity between a verb (e.g., chew) and verb-particle (e.g., chew out). They found that this relationship influenced lexical decision performance in an on-line masked priming task, with facilitation of semantically similar verb/verb-particle pairs (see Appendix A for sample similarity ratings and Appendix B for priming results).

Gonnerman and Hayes (2005) also demonstrated that the ordering preferences found in corpora were reflected in the online processing of sentences varying the lexical and syntactic factors of adjacency, dependency, and NP length in verb-particle constructions. Results showed that reading times increased for shifted sentences, highly dependent constructions, and sentences with long intervening NP's. Consistent with the corpus studies of Hawkins (2004) and Lohse et al. (2004), Gonnerman and Hayes showed that shifting the particle allows the language user to recognize all sentence constituents earlier. Thus, the NP is recognized immediately after the verb. However, they found that at times minimization of the semantic domain can work against syntactic minimization. If the verb is dependent on the particle for its interpretation, it cannot be fully processed until the particle is encountered. This tends to push users to position the particle next to the verb in order to minimize the semantic domain. Therefore, there are competing forces that interact to sometimes make processing easier if the particle is adjacent to the verb and sometimes easier when shifted. 
The results from both the corpora and experimental comprehension studies support the notion of word order preferences reflecting the minimization of syntactic and semantic domains. However, the selfpaced reading study also demonstrates how these semantic and syntactic factors interact in a probabilistic fashion to influence processing and indicate that when different performance factors interact, the syntactic and semantic domains can compete for processing efficiency.

\section{Adjacency, Dependency, and Complexity in Verb-Particle Constructions}

The present study examines the interaction of several factors that individually have been shown to affect sentence processing: constituent adjacency, dependency between phrasal elements, and relative clause complexity (c.f. Keenan \& Hawkins, 1987; Lohse et al., 2004; Gonnerman and Hayes, 2005). By varying all three factors within a given sentence, I will be able to examine competing forces on processing efficiency, and to clarify which factors maximize processing when several factors interact in one sentence, especially when the factors act in opposition to one another.

In verb-particle constructions, the manipulation of the adjacency of verb and particle affects whether the syntactic or semantic domain is minimized, with adjacent verb and particle facilitating semantic processing and shifted particle facilitating syntactic processing via earlier constituent recognition.

Manipulating the dependency relationship between verb and particle influences the relative importance of adjacency on processing. Adjacency of the verb and particle facilitates processing of the semantic domain, especially when the verb and particle are dependent on each other for their meaning. When dependency is low, I am interested to see whether the minimization of the syntactic domain is preferred over the semantic.

Finally, changing the complexity of the direct object NP should reveal how structures which are

more common across languages of the world affect processing within sentences. I expect that increasing the complexity of the relative clauses will have different effects on processing based on the position of the particle and the dependency of the verb and particle. That is, increased complexity should have its greatest effect when the verb and particle are shifted, especially when the verb and particle are highly dependent. This ordering requires the integration of a dependency relationship across a complex clause, which according to Gibson (2000) should increase working memory load, thus increasing processing difficulty. However, when the particle is adjacent, the dependency relationship can be resolved before encountering the complex relative clause. 


\subsection{Self-paced Reading of Verb-Particle Constructions}

In order to determine how processing in individuals is affected by the combination of several factors that have each been shown to influence processing independently and also underlie the distribution of grammaticalized structures across languages, I varied the following factors in a word by word self-paced reading task: adjacency, dependency, and noun phrase complexity.

\subsubsection{Method}

\subsubsection{Participants}

63 undergraduates participated for course credit. All were native speakers of English.

\subsubsection{Materials}

Each sentence began with a two-word subject NP (e.g., the man; the teacher) that was controlled for frequency across conditions. All of the verb phrases were in the future tense (e.g., The man will look up...) in order to avoid irregular conjugations.

The verb phrases for the target sentences were based on 78 verb-particle constructions that varied in three levels of dependency: 26 low dependency (e.g., finish up); 26 middle dependency (e.g., look up); and 26 high dependency (e.g., chew out). Dependency was determined by both a similarity judgment task and a masked priming task (Gonnerman \& Hayes, 2005). For summaries of the results from the survey and priming task see Appendices A and B, respectively.

For each verb-particle construction, four direct object NPs, varying in complexity, were created. Different types of sentences contained either no relative clause or a relative clause of increasing complexity (subject, object, or genitive) since the complexity of relative clauses has been shown to affect processing ease (Keenan \& Hakwins, 1987). Thus, the complexity variable consisted of four sentences of the same length (5 words): no relative clause (e.g., the class of disruptive students); subject relative (e.g., the class who always cheated); object relative (e.g., the class that teachers hated); and genitive relative (e.g., the class whose teacher fainted).

In addition, two versions of each sentence were created for each verb-particle construction and each type of relative clause, one with the verb and particle adjacent, and one with the particle placed after the direct object NP.

Finally, plausible phrases were added to the end of the sentences to prevent wrap-up effects on the last target word of each sentence, for example, 'next week' in the sentence: 'The boss will smooth the difficult and troubling problem over next week.' 
Overall, eight sentences were created for each verb-particle construction, reflecting the four levels of complexity and two levels of adjacency (see Table 1 for a sample set of sentences), for a total of 624 target sentences. These sentences were divided into 8 lists, such that participants only saw one sentence for each verb-particle construction, that is, 78 total target sentences. 234 filler sentences of similar length to those of the target sentences were added to each list, for a total of 312 sentences per list.

Table 1: Sample set of sentences for the verb-particle construction blow off (high dependency).

\begin{tabular}{ccc}
\hline Relative Clause Type & Adjacency & Sample Sentence \\
\hline None & Adjacent & The boy will blow off his boring American history class. \\
None & Shifted & The boy will blow his boring American history class off. \\
Subject Relative & Adjacent & The boy will blow off his class that lasts forever. \\
Subject Relative & Shifted & The boy will blow his class that lasts forever off. \\
Object Relative & Adjacent & The boy will blow off his class that Johnson teaches. \\
Object Relative & Shifted & The boy will blow his class that Johnson teaches off. \\
Genitive Relative & Adjacent & The boy will blow off his class whose teacher rambles. \\
Genitive Relative & Shifted & The boy will blow his class whose teacher rambles off. \\
\hline
\end{tabular}

\subsubsection{Procedure}

Participants were tested individually in a sound-attenuated room. Sentences were presented one word at a time on a computer screen, with black letters on a white background. Participants read at their own pace, pressing a button to replace the word they had just read with dashes and to display the next word of the sentence. Participants were given several practice items before beginning the test sentences. After reading each sentence, participants answered a yes-no content question to ensure careful reading. Reading times for each button press were recorded. Testing sessions generally lasted twenty minutes.

\subsubsection{Results and Discussion}

\subsubsection{Mean Reading Times}

Reading times for sentences in which participants answered the yes-no question incorrectly were excluded from analysis. In addition, individual word reading times below $100 \mathrm{msec}$ or above 1000 msec were excluded from the latency analyses.

Mean reading times per word were calculated for each sentence. These values were then entered into an ANOVA with the factors of Adjacency (adjacent, shifted), Dependency (low, middle, high) and Complexity (no relative, subject relative, object relative, genitive relative). See Table 2 for mean reading 
times by condition. There were main effects of Adjacency: $F(1,62)=4.1, p<0.05$, with shifted sentences taking longer to read on average (387 msec) than adjacent ones (381 msec); Dependency: F (2, $124)=6.0, p<0.01$, with reading times increasing as the verb becomes more dependent on the particle for its meaning, except in the high dependency condition in which reading times decreased; and Complexity: $\mathrm{F}(3,186)=5.7, \mathrm{p}<0.001$, with sentences containing more complex direct object NP's taking longer to read (see Figure 1).

Table 2: Mean reading times (msec) by Complexity (no relative, subject, object, genitive relatives) and Dependency (low, middle, high) for sentences with adjacent and shifted particles.

\begin{tabular}{cccccccc}
\hline & \multicolumn{7}{c}{ Dependency } \\
\cline { 2 - 8 } & \multicolumn{2}{c}{ Low } & \multicolumn{2}{c}{ Mid } & \multicolumn{2}{c}{ High } \\
\hline Complexity & Adj & Shift & Adj & Shift & Adj & Shift & Mean \\
\hline No Relative & 338.1 & 384.4 & 383.1 & 380.3 & 410.6 & 378.3 & $\mathbf{3 7 9 . 1}$ \\
Subject Relative & 387.2 & 362.7 & 377.0 & 384.1 & 369.6 & 383.3 & $\mathbf{3 7 7 . 3}$ \\
Object Relative & 390.0 & 357.9 & 399.6 & 405.0 & 367.0 & 399.9 & $\mathbf{3 8 6 . 6}$ \\
Genitive Relative & 383.3 & 415.4 & 389.6 & 394.5 & 378.7 & 408.2 & $\mathbf{3 9 5 . 0}$ \\
\hline Mean & $\mathbf{3 7 7 . 4}$ & $\mathbf{3 8 9 . 1}$ & $\mathbf{3 8 6 . 9}$ & \\
\hline
\end{tabular}

The main effects indicate that more processing is required, as reflected in increased reading times, for sentences with complex direct object NPs (e.g., object and genitive relatives) and shifted particles. It is unclear why reading times decreased for sentences with high dependency verb-particle constructions. These high dependency verb-particle constructions will be explored more in the following plausibility analysis.

There was an interaction of Adjacency by Dependency: F $(2,124)=20.3, p<0.001$, with reading times increasing as dependency increased, but, only in shifted sentences (see Figure 2). The interaction between Adjacency and Dependency showed slower reading times for shifted, high dependency sentences (The principal will chew the class who always cheated out). That is, it took longer to read words in a sentence when a highly dependent particle was shifted away from its verb, regardless of the complexity of the intervening direct object NP. 


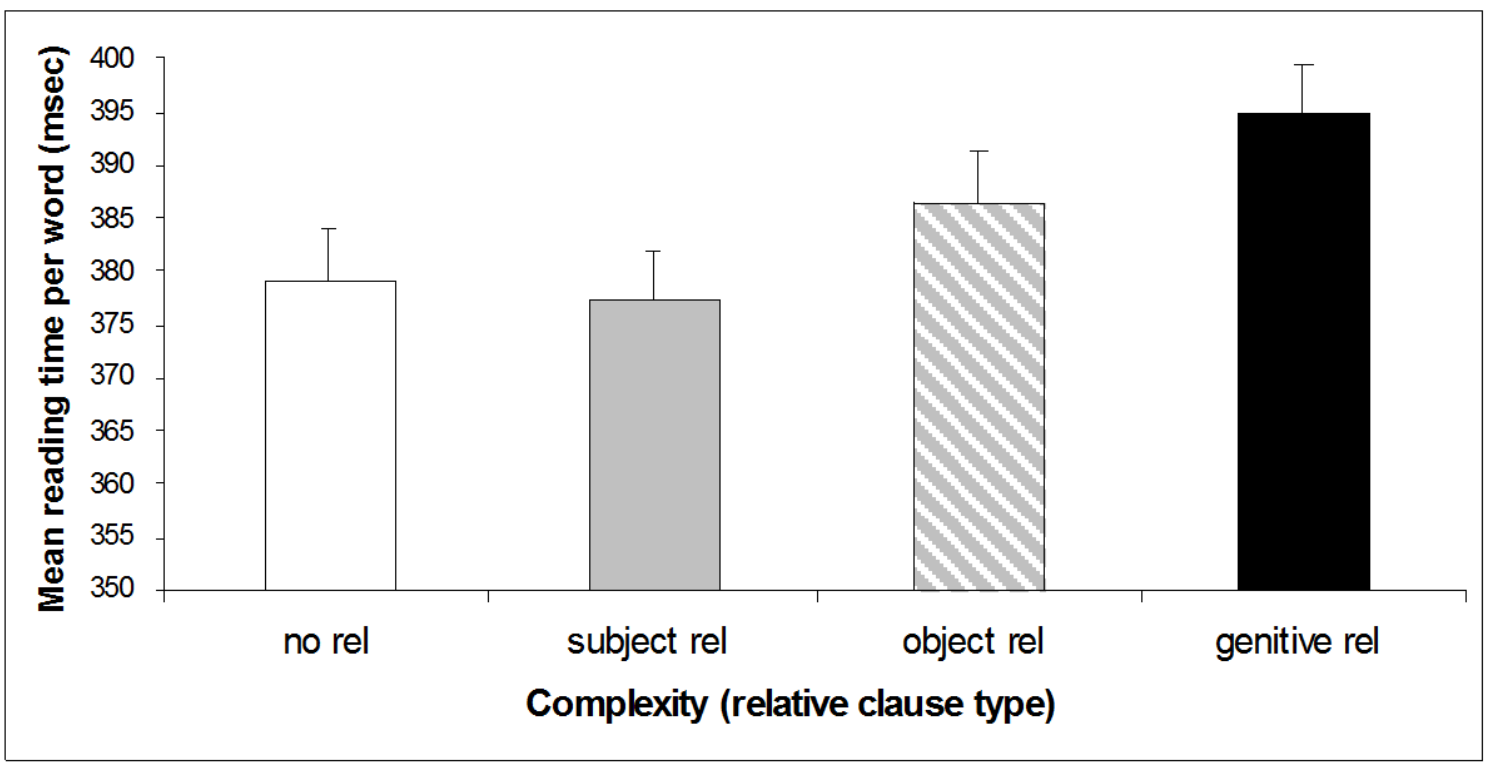

Figure 1. Mean reading times per word (msec) for sentences varying in complexity: no relative clause, subject, object, or genitive relative clauses.

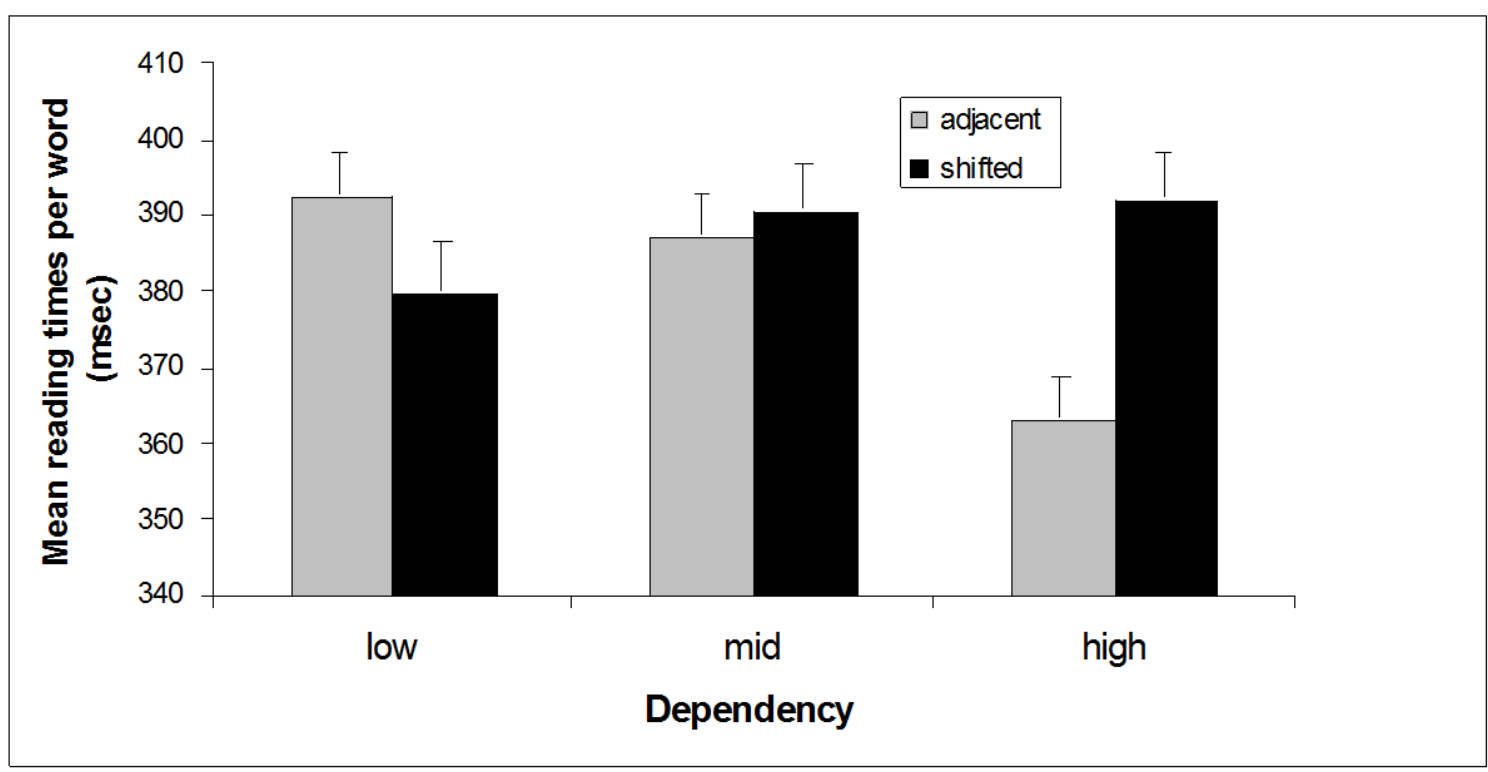

Figure 2. Mean reading times per word (msec) for adjacent and shifted sentences with low, mid, or high dependency verb-particle constructions.

There was also an interaction of Adjacency by Complexity: F $(3,186)=3.3, \mathrm{p}<0.05$, with slower reading times for more complex NPs (object and genitive relatives), in shifted sentences (e.g., The teacher will line her students whose parents arrived up). It was more difficult to read sentences when a complex NP intervened between the verb and particle, regardless of the degree of dependency between the verb and particle (see Figure 3). 


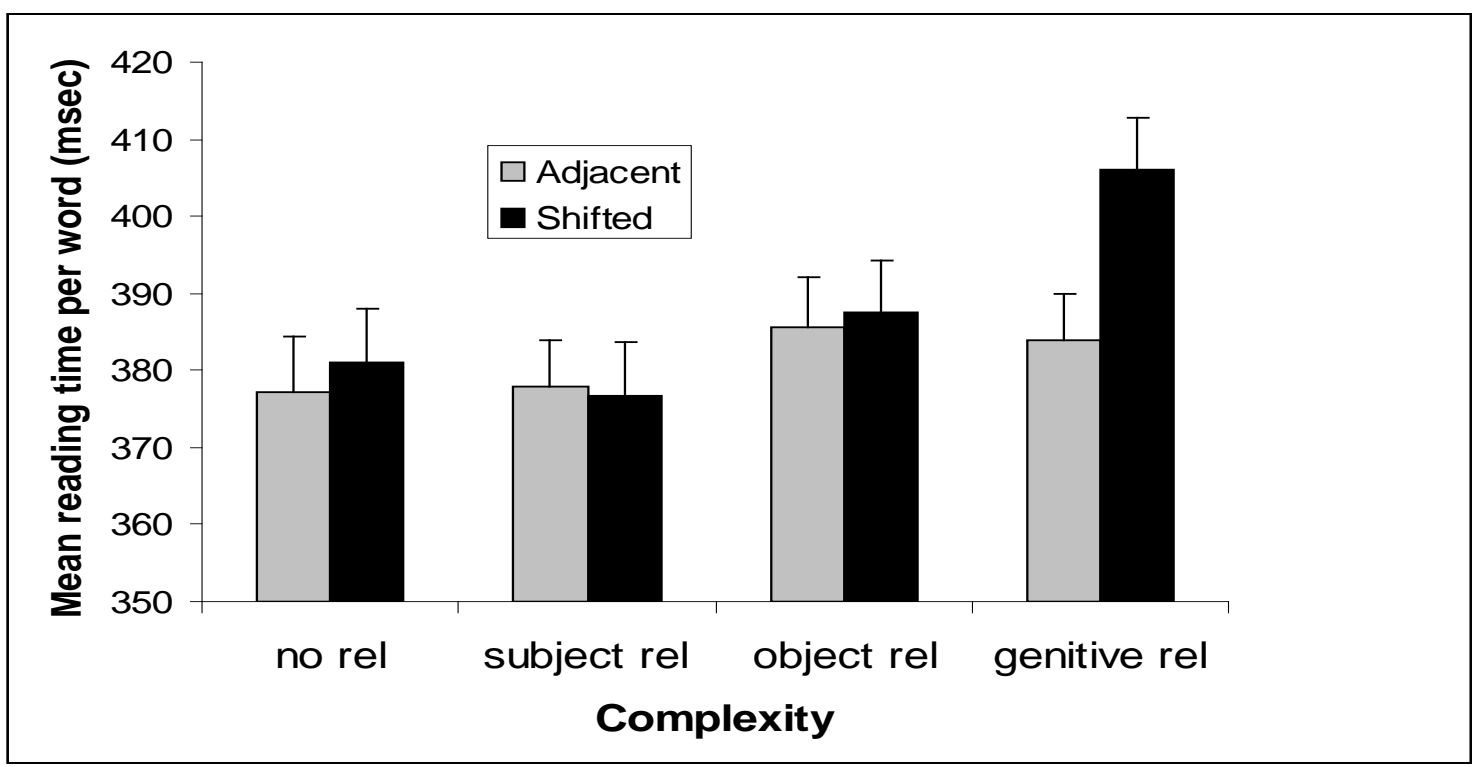

Figure 3. Mean reading times per word (msec) for adjacent and shifted sentences with no relative clause, subject, object, or genitive relative clauses.

There was an interaction of Complexity by Dependency: F $(6,372)=6.4, \mathrm{p}<0.001$, which showed that reading times generally increased as dependency increased. However, this effect was only seen for the no relative, subject relative, and object relative direct object NPs. Reading times were consistently slow for the genitive relatives, regardless of dependency. This result suggests ceiling effects for NP complexity, indicating that when sentences contain very complex direct object NPs (genitive relatives), increasing the dependency relationship between verb and particle (e.g., chew out) cannot increase reading times any further. However, when the direct object NPs are less complex (i.e., no relatives, subject relatives, or object relatives) a graded effect of verb-particle dependency was seen, that is, reading times increased when a sentence contained a more dependent verb-particle (e.g., chew out) versus a less dependent verb-particle (e.g., finish up) (see Figure 4).

Finally, there was a three-way interaction of Adjacency, Complexity, and Dependency: F (6, 372) = 3.3, $\mathrm{p}<0.01$, with reading times increasing in the shifted sentences, but only for sentences containing more complex relative clauses and dependent verb-particles (see Figures 5 and 6 for graphs of adjacent and shifted conditions, respectively). 


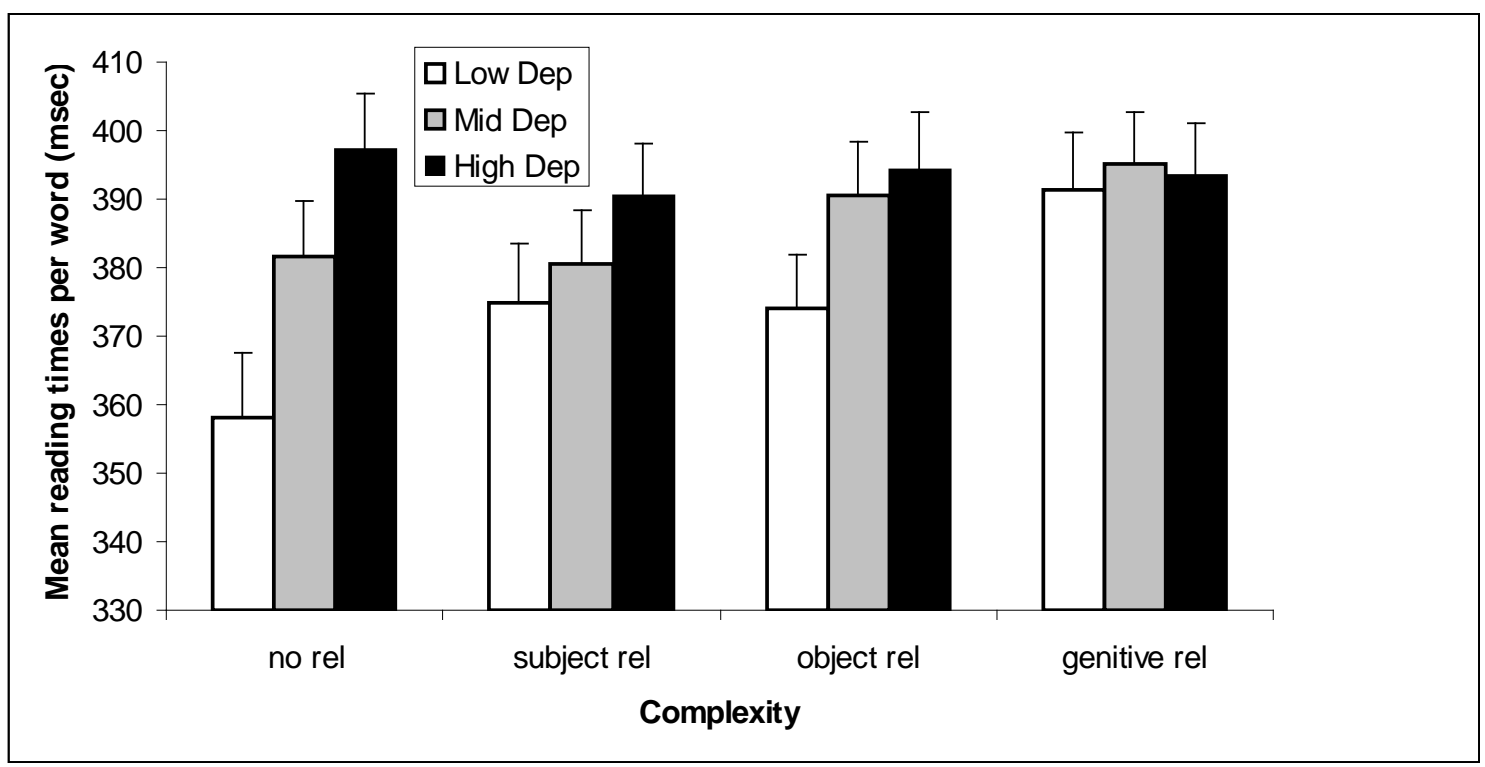

Figure 4: Mean reading times per word (msec) for sentences with no relative clause, subject, object, or genitive relative clauses, and low, mid, or high dependency verb-particle constructions.

Across levels of Adjacency, the interaction of Complexity and Dependency showed an increase in reading times for sentences containing shifted particles (see Figure 6), but only for subject and object relatives with mid and high dependency verb particles and genitive relatives regardless of dependency. These results indicate an overload of processing resources when complex NPs occur between more dependent verbs and particles. However, processing also slows for low dependency verb-particles when a genitive relative intervenes between verb and particle, suggesting that genitive relatives tax processing resources regardless of the semantic dependency relationship between verb and particle.

The graded effect of dependency for sentences containing subject and object relative clauses showed an opposite pattern when the particle was adjacent to its verb versus when it is shifted away from its verb. As stated earlier, in particle shifted sentences, reading times increased as dependency increased, suggesting that it is more difficult to read sentences with complex NPs, especially when a dependent particle is shifted away from its verb (e.g., the principal will chew the class who always cheated out). Unexpectedly, however, in particle adjacent sentences, reading times generally decrease as dependency increases (see Figure 5). This could be due to the fact that the verb phrase is completely interpreted when the verb and particle occur adjacently (e.g., the principal will chew out the class who always cheated). The effect of dependency is reduced, since higher dependency verb-particle constructions benefit from an adjacent interpretation and can then be processed more easily. 


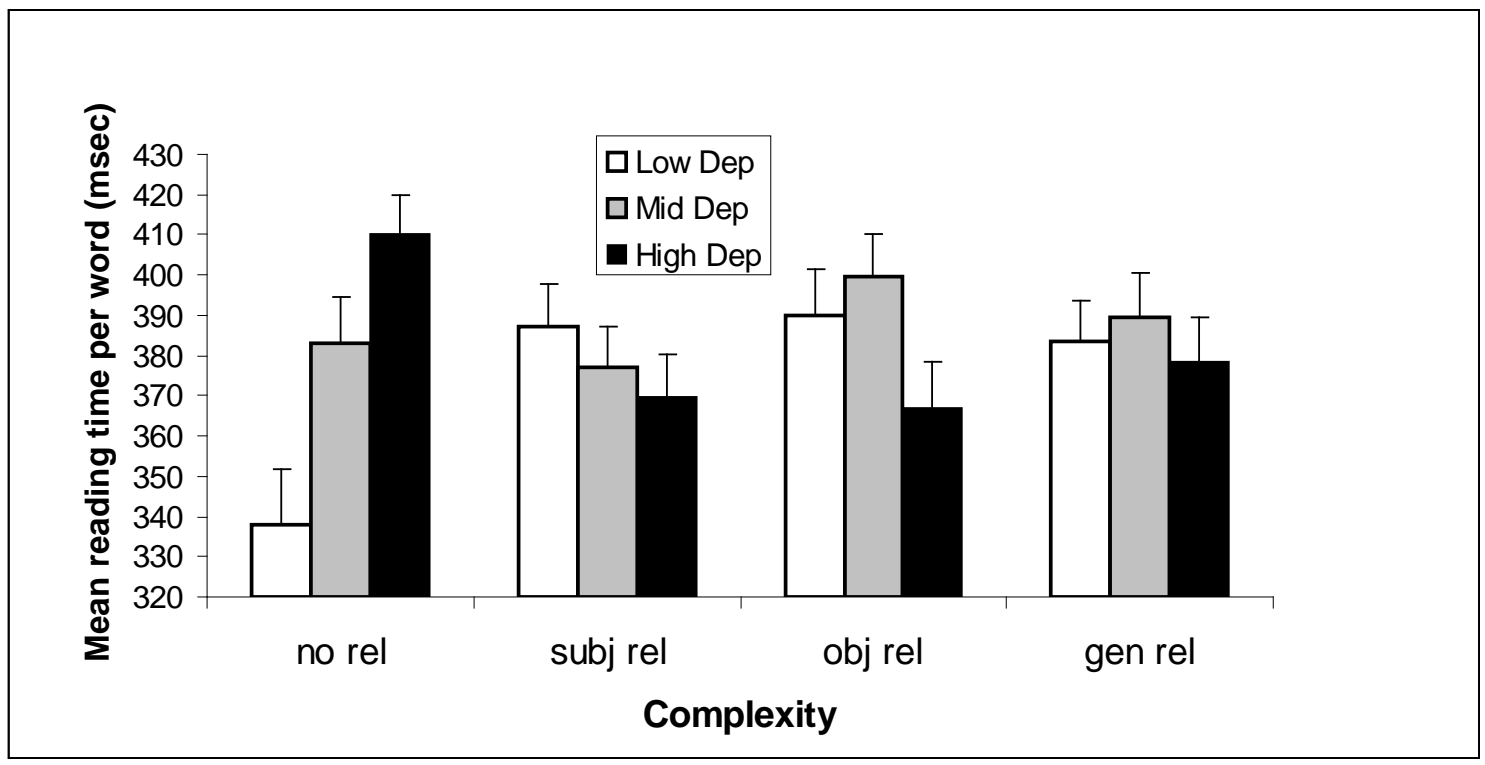

Figure 5: Mean reading times per word (msec) for particle adjacent sentences with no relative clause, subject, object, or genitive relative clauses, and low, mid, or high Dependency verb-particle constructions.

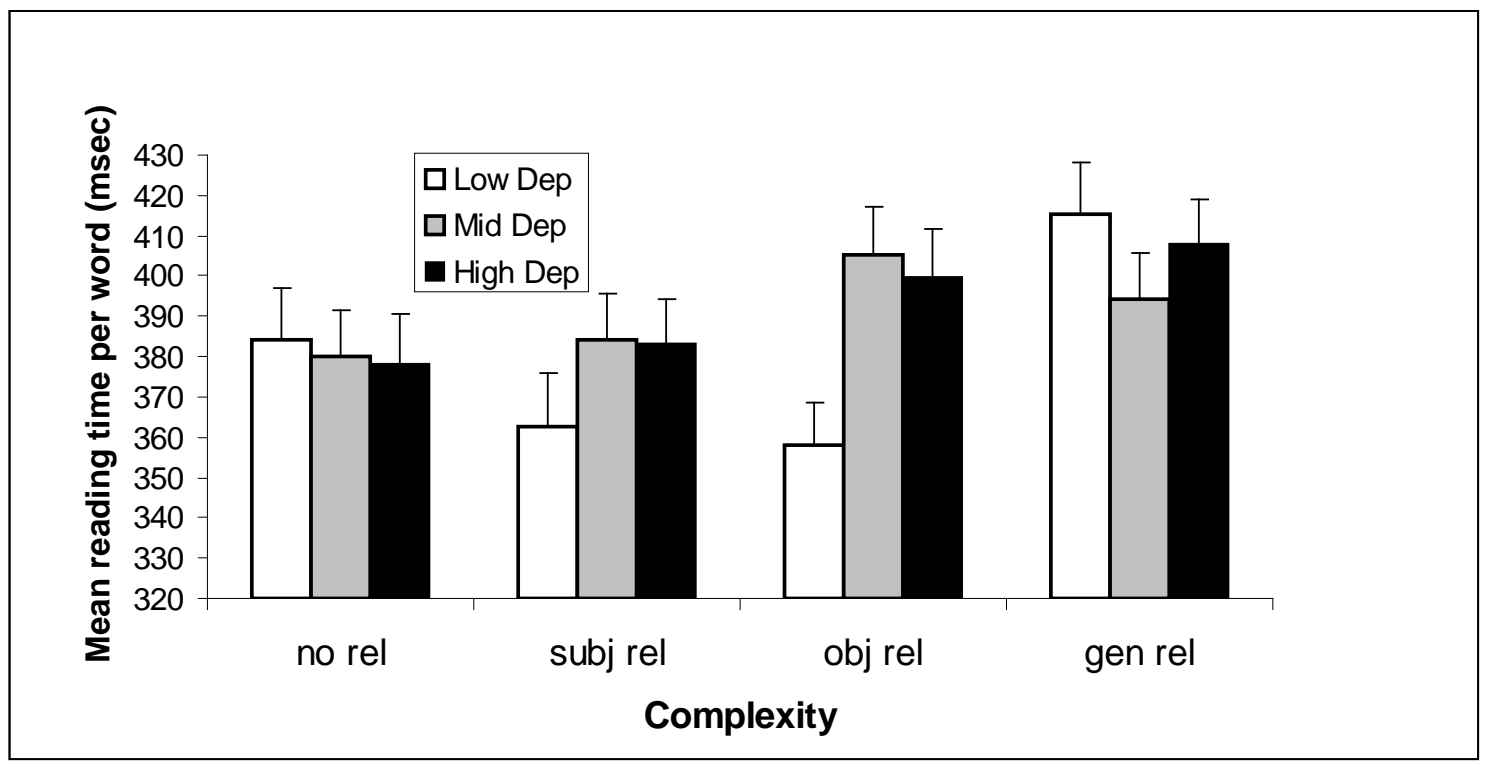

Figure 6: Mean reading times per word (msec) for particle shifted sentences with no relative clause, subject, object, or genitive relative clauses, and low, mid, or high Dependency verb-particle constructions.

An unusual effect was also found for sentences with no relative clauses as the direct object NP. There was a large, increasing, effect of dependency in particle adjacent sentences, but almost no effect of dependency in the shifted sentences. The effect for sentences with no relative clauses was mostly driven by a very slow mean reading time $(410 \mathrm{msec})$ for words in the adjacent, high dependency condition (e.g., the principal will chew out the class of disruptive students). It is unclear why reading times are so slow for 
this condition for which I expected some of the fastest reading times (because the complexity of the direct object NP was so low). It is possible that this unusual result was due to the fact that these were mean reading times per word. A word by word analysis was conducted to better understand the trends underlying these results.

\subsubsection{Word by word analysis}

To determine where the patterns of reading times occurred in each sentence, mean word by word reading times were analyzed for sentences according to type of relative clause for adjacent (Figure 7) and shifted verb-particle constructions (Figure 8). The interaction of Adjacency by Complexity was significant: F $(3,186)=3.3, p<0.05$, with reading times increasing for sentences with more complex direct object NPs, especially in the particle shifted sentences (e.g., teacher will cross the writing that looked messy out).

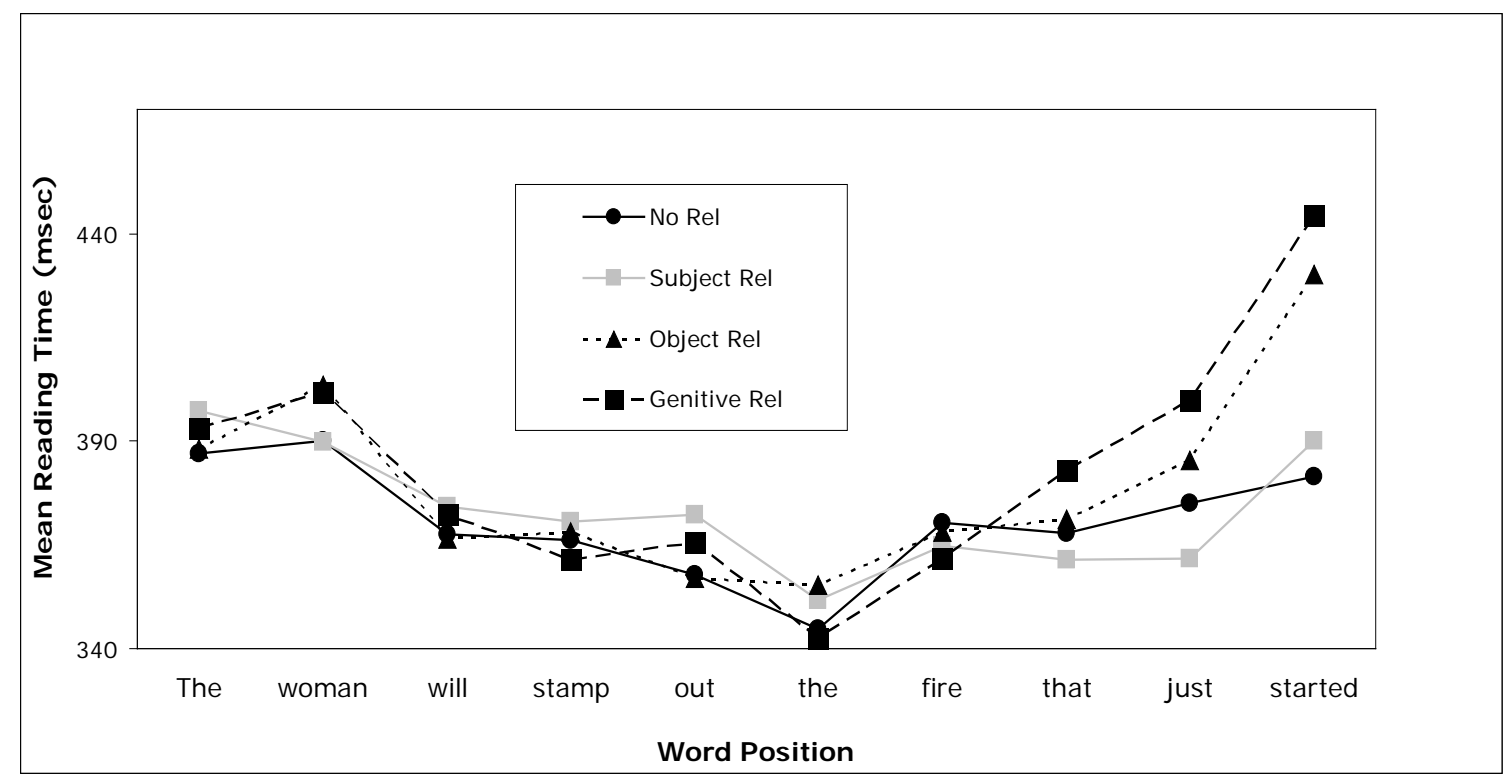

Figure 7: Mean word by word reading times by complexity for sentences with verb and particle adjacent. 


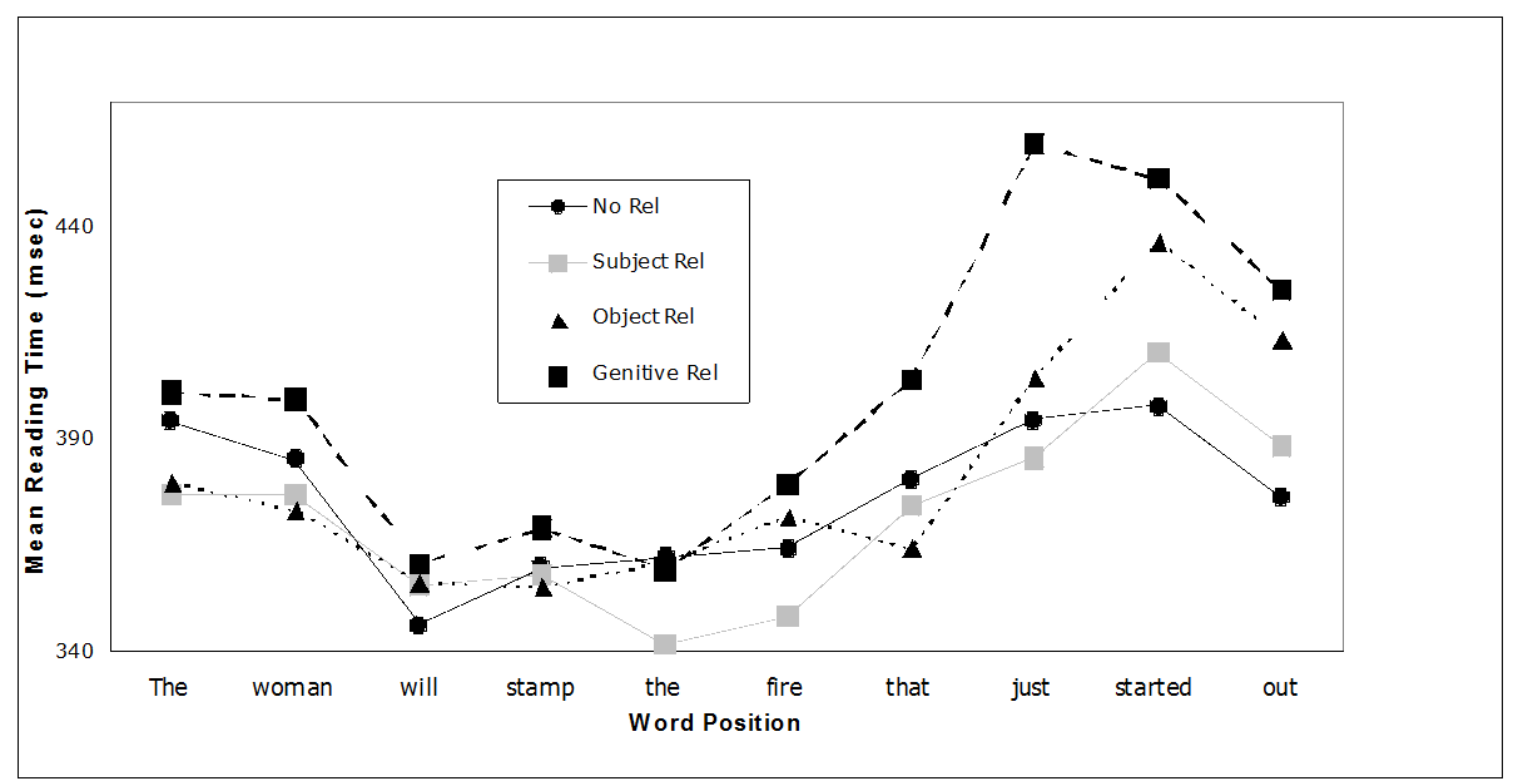

Figure 8: Mean word by word reading times by complexity for sentences with the particle shifted away from the verb.

As expected the pattern of results was similar between the adjacent (15) and shifted (16) sentences until the head noun of the direct object NP was encountered in the shifted sentences. For example, 'fire' in sentence (16) below:

(15) The wife will stamp out the fire that just started.

(16) The wife will stamp the fire that just started out.

At this point reading times were slower in the shifted sentences. This is most likely due to the tendency for the head noun to be interpreted as the direct object of the verb without the particle. When the verb is dependent on the particle for its meaning, interpreting the NP as the direct object of the verb alone can be misleading, as in, 'The principle will chew the students'. The increase in reading times for the head noun is not seen in adjacent sentences because the head noun is not misinterpreted when the verb and particle occur together, as in, 'The teacher will chew out the students'. I will examine the plausibility of the head noun as the direct object of the verb alone in the section 'Analysis of Plausibility'.

Another trend demonstrated by the word by word analysis is the graded increase in reading times for the entire direct object NP as complexity increased. That is, direct object NP's with genitive relatives took longer to read than direct object NP's containing less complex relatives. This effect was exaggerated in the shifted sentences, such that reading times were even slower for the more complex NPs when the particle was shifted to the end of the sentence.

\subsubsection{Particle reading times}

The word by word analysis demonstrated that when the particle occurred adjacent to the verb, reading 
times for the particle were fairly fast and remained fairly consistent across levels of complexity. Yet, when the particle was shifted away from the verb, reading times on the particle increased dramatically and were graded according to NP complexity.

In order to show how reading times for the particle varied by dependency, complexity, and adjacency; mean differences in reading times for the particle in the shifted and adjacent positions were calculated (see Figure 9). Reading times for the particle in the shifted position increased with increasing complexity for low dependency verbs (e.g., the shifted particle 'up' in 'finish [direct object NP] up' took longer to read when the intervening direct object NP was a genitive versus a subject relative). Interestingly, when the NP did not contain a relative clause, reading times for the shifted particle were faster than the adjacent. This effect illustrates one case in which shifted structures are preferred over the adjacent. This effect supports the notion that minimization of the syntactic domain is preferred, but only when the semantic relationship between verb and particle is low and the NP is of low complexity.

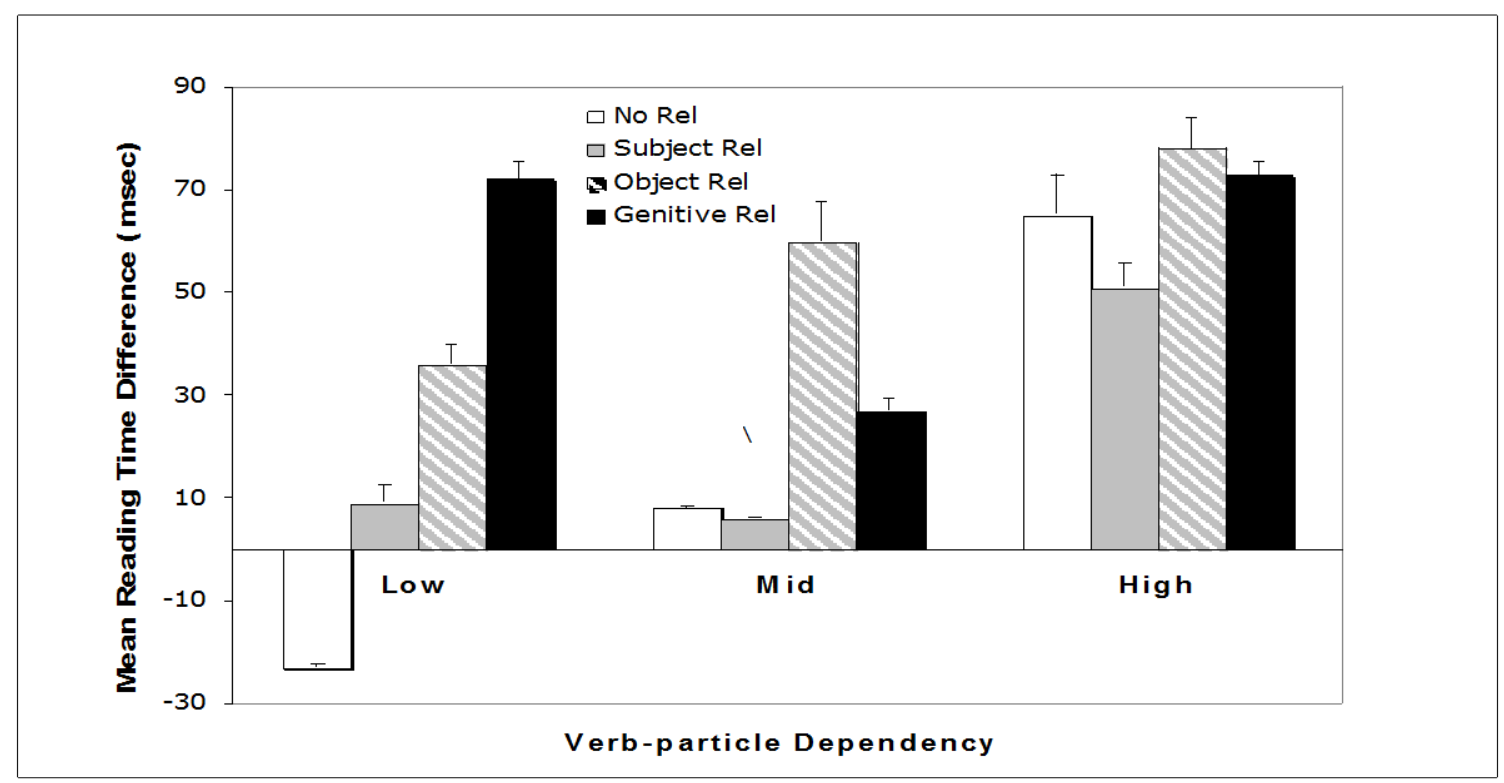

Figure 9: Mean reading time differences (msec) for the particle (shifted - adjacent) according to verb-particle dependency and direct object NP complexity.

For high dependency verbs, reading times were slow in the shifted position for all NP types. For example, when a sentence contained a high dependency verb-particle such as 'chew out,' reading times were slower for 'out' when it was shifted regardless of the complexity of the intervening NP. For middle dependency verbs (e.g., look up), reading times for the shifted particle (e.g., up) increased only for the most complex NP's, that is, object and genitive relatives.

Results showed that reading times for the particle alone are affected by adjacency, dependency, and 
complexity. The most straightforward complexity effect occurred for low dependency verbs, with reading times for the shifted particle increasing as complexity increased. However, for the high dependency pairs, shifting caused longer reading times for the particle, regardless of the complexity of the direct object NP. This pattern suggests that when processing is made sufficiently difficult (as in shifted, high dependency sentences) complexity does little to increase the difficulty. For example, 'The principal will chew the class of disruptive students out,' which has no relative clause is almost as difficult to process as 'The principal will chew the class whose teacher fainted out,' which has a high complexity NP. However, when sentences require fewer processing resources (as in low dependency conditions), the complexity of the intervening direct object NP has a greater effect on reading times for the shifted particle.

\subsection{Analysis of Plausibility}

The word by word analysis of the sentences revealed that adjacency affects reading times for both the head noun in the direct object NP and for the particle. When a particle is shifted, an ambiguity can arise, where the direct object NP is interpreted as the direct object of the verb alone. Sometimes this NP is a plausible direct object for the verb without the particle and sometimes it is not. For example, "the students" is implausible as the direct object in the sentence, “The teacher will chew the students out," until the reader encounters the particle. I conducted a survey to determine whether the NP's were plausible as direct objects of the verbs alone, without their particles.

\subsubsection{Method}

\subsubsection{Participants}

203 undergraduate students participated for course credit. All were native speakers of English. None of the participants were involved in the self-paced reading study.

\subsubsection{Materials}

The 78 verbs (without their particles) and head nouns from the self-paced reading experiment's target sentences were used in the plausibility survey. The sentences were presented such that the direct object noun phrase only consisted of the determiner and head noun (e.g., the problem, the candy, the class). The direct object NPs were always placed adjacent to the verbs and the particles were not included in the sentences (e.g., the man will buy the stores or the principal will chew the students).

These shortened sentences were divided into 2 lists, each containing 39 sentences. Levels of dependency were evenly distributed in each survey, such that 13 low, middle, and high dependency verbs occurred in each survey. See Table 3 for a set of sample stimuli. 
Table 3: Sample set of stimuli for the plausibility survey

\begin{tabular}{cc}
\hline Dependency & Sample Sentence \\
\hline Low & The children will finish their meal. \\
Low & The students will clear the floor. \\
Middle & The mother will take her promise. \\
Middle & The man will look the word. \\
High & The man will string the woman. \\
High & The principal will chew the class. \\
\hline
\end{tabular}

\subsubsection{Procedure}

Participants were asked to rate the plausibility of each sentence (i.e., how much the sentence 'makes sense') on a scale from 1 (very implausible) to 9 (very plausible). The instructions included examples of plausible as well as implausible sentences with corresponding ratings.

\subsubsection{Results and Discussion}

Average plausibility ratings were calculated for each sentence. These ratings were then entered into an ANOVA with the factor of Dependency (low, middle, high). The effect of Dependency was significant: F $(2,75)=7.1, \mathrm{p}<0.01$, with a graded effect of dependency. That is, sentences containing low dependency verbs (e.g., the waiter will bring the wine) were rated as the most plausible (mean rating 6.81). Sentences including high dependency verbs (e.g., the man will string the woman) were rated as least plausible (mean rating 4.96). Finally, sentences with middle dependency verbs (e.g., the mother will take her promise) were rated in between that of the low and high dependency verbs (mean rating 5.51).

The dependency effect confirms our prediction that the verbs in low dependency verb-particle constructions do not depend highly on their particles for their meaning and are thus more plausible than verb-particle pairs that are dependent. For example, the meaning of the sentence with a low dependency verb-particle, 'the children will finish their meal up,' is very similar and just as plausible as 'the children will finish their meal,' which does not contain the particle. Conversely, a sentence with a middle or high dependency verb-particle, 'the tutor will catch the student up,' means something very different with the particle removed as in, 'the tutor will catch the student' or is simply implausible without the particle (e.g., 'the principal will chew the class' versus 'the principal will chew the class out').

\subsection{Plausibility Analysis of Reading Times}

Using the plausibility ratings discussed in the previous section, the sentence stimuli from the self-paced reading task were divided into high and low plausibility conditions. Sentences rated 5.0 and above on the 
plausibility survey were included in the high plausibility condition, and those rated below 5.0 were included in the low plausibility condition.

The reading times for the particle and the head noun were analyzed by dependency and plausibility. For high dependency verb-particles in sentences categorized as 'low plausibility', reading times were similar for the adjacent and shifted particle (see Figure 10). For example, the particle 'out' was read just as fast in the shifted position, 'the professor will chew the students out' as it was in the adjacent "the professor will chew out the students.' This result was surprising given that I expected reading times for the shifted particle to be slower than that of the adjacent particle. This effect is possibly due to readers' expectation of the particle, since the verb and head noun alone are uninterpretable. For example, when reading the sentence 'the man will string the woman,' the listener may realize that 'woman' is an implausible direct object for the sentence. This may create an expectation that additional information is coming to make the previous section of the sentence plausible. In line with this hypothesis, I found that reading times for the head noun (e.g., woman) was much slower when the particle was shifted in a low plausibility sentence (see Figure 11). Reading times for the shifted head noun itself are slow because the noun is not understandable as the object of the verb until the particle is read (e.g., The principal will chew the class of disruptive students out). The increase in reading time for the head noun may indicate that this is the point at which processing is most difficult, perhaps because the head noun is implausible and additional information is expected. According to Lewis and Nakayama (2002), this expectation should reduce processing on the shifted particle, thus decreasing reading times due to correspondence between what is expected and what is read.

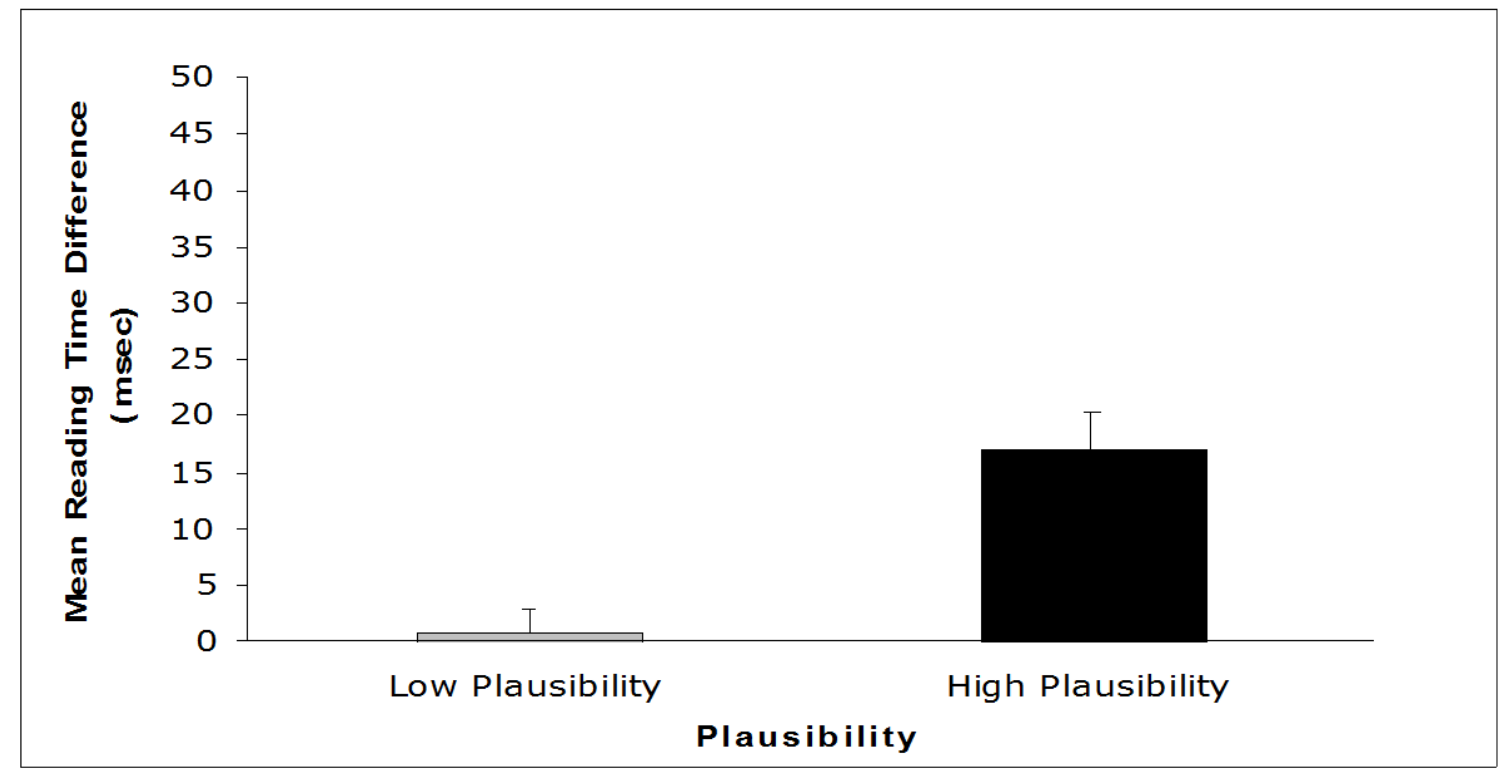

Figure 10: Mean reading time difference (msec) for high dependency verb-particles by plausibility. 
In the high plausibility condition, reading times were slower for the shifted particle than the adjacent particle (see Figure 10). The slow down for the particle may be due to reanalysis of the sentence at the shifted particle, for example, 'the man will throw the food' will need to be reanalyzed when the particle is read, as in 'the man will throw the food up.' In contrast with the low plausibility condition, this suggests that processing difficulty increases only at the shifted particle. This would explain why reading times only increased at this particle and not the head noun, since the head noun in these sentences is a plausible direct object (e.g, the man will throw the food). Consistent with this hypothesis, reading times for head nouns were faster in this high plausibility condition (see Figure 11).

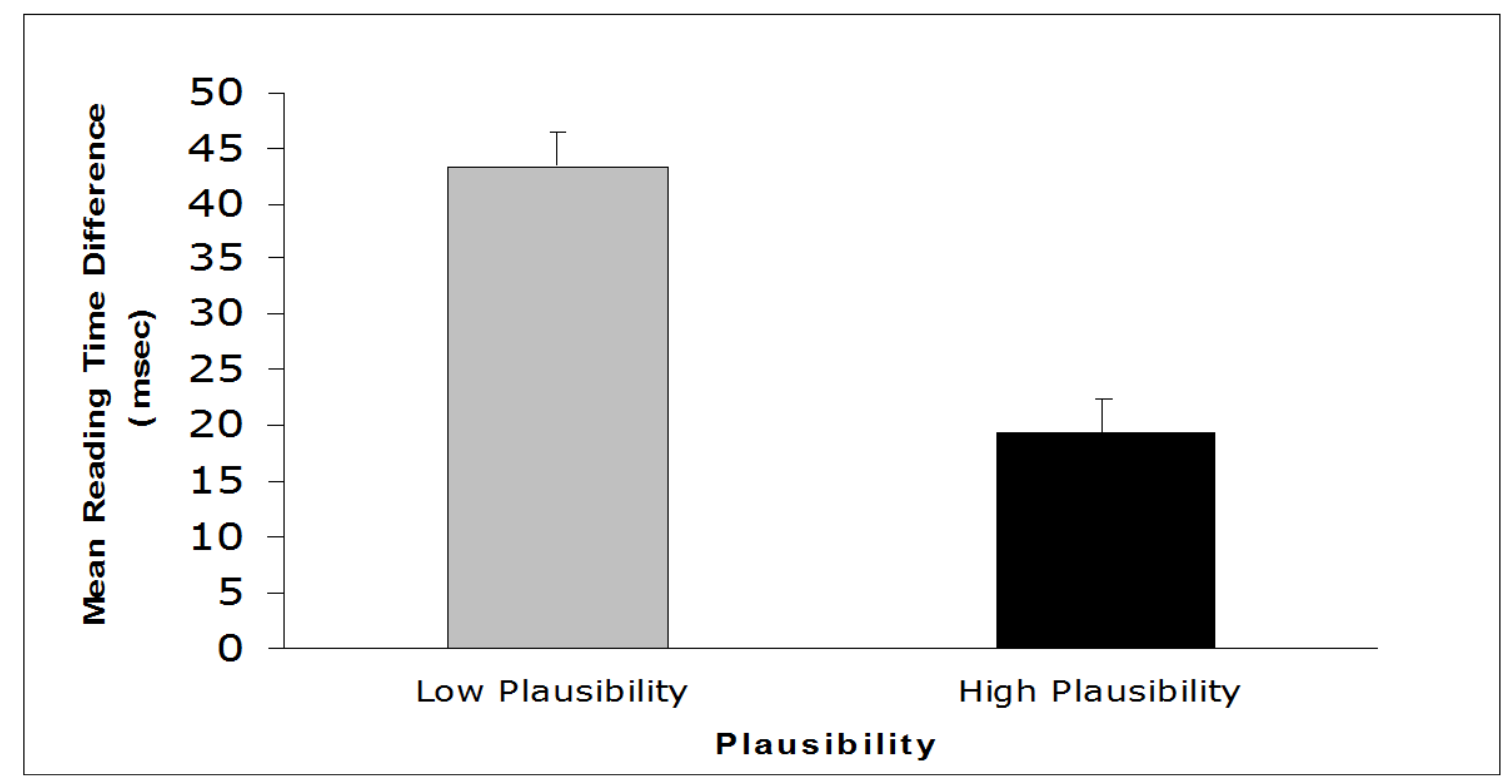

Figure 11: Mean reading time difference (msec) for head nouns in sentences with high dependency verb-particle constructions according to plausibility.

\section{Overview of Results}

The results from the self-paced reading task confirmed the predictions that adjacency of the verb and particle plays a major role in sentence comprehension. The tendency to read shifted sentences more slowly was only exacerbated by the inclusion of more dependent verb-particle relationships and more complex direct object NP's. However, when both dependency and NP complexity were low (i.e., no relative clause and a low dependency relationship), participants read the shifted particles faster (see Figure 9).

More specifically, results indicated that it is more difficult to process shifted sentences with more complex intervening noun phrases. Results showed that sentences with genitive relatives resulted in slower reading times than those with direct objects which were, in turn, were read slower than sentences with subject relatives, especially when these NP’s intervened between the verb and particle. 
Results also showed that the average reading times slowed as dependency increased when the particle was shifted. However, when the particle was adjacent, reading times got slightly faster as dependency increased.

There was a clear effect of dependency when the complexity of the relative clauses was low (i.e., no relative, subject, and object). However, ceiling effects were found for the genitive relatives. When examined by adjacency, both increased complexity and dependency resulted in slower reading times when the particle was shifted, but not when it was adjacent.

The trends revealed in the word by word analysis confirmed the average reading time results, but more clearly illustrated the impact of NP complexity. In shifted sentences, word by word reading times across the intervening direct object NP increased dramatically as complexity increased. When the verb and particle were adjacent, the complexity effect was almost non-existent.

Reading times on the particle itself varied by adjacency, dependency, and complexity. When dependency was low, there was a graded effect of complexity, with the shifted particle read faster than the adjacent when complexity was low, but read slower as complexity increased. For high dependency items, reading times for the shifted particle were slow across complexity indicating ceiling effects when the verb and particle are separated, but highly dependent.

Results from the plausibility survey demonstrated that the dependency of verb-particles is related to the plausibility of the head noun when the particle is shifted. Findings showed that high dependency verbparticles were strongly affected by plausibility, such that for plausible head nouns, reading times on the shifted particle increased (suggesting reanalysis) and for non-plausible head nouns, the noun itself was read more slowly, while the particle was read faster.

\section{General Discussion}

The inclusion of the three factors of adjacency, dependency, and complexity allowed for an examination of the interaction of opposing forces on processing, that is, the interaction of the syntactic and semantic domains. According to Hawkins' theory, shifted particles minimize syntactic domains since the direct object NP can be recognized earlier if the particle is shifted to the end of the sentence compared to when the particle occurs adjacent to the verb. For example, only 5 words are required to recognize the final constituent (the direct object NP) in the sentence, 'the boy will look the number up,' but 6 are required to recognize the direct object NP in the sentence, 'the boy will look up the number.' However, results from this study indicate that sentences in which the particle occurred adjacent to the verb were processed more easily, except for faster reading times of the shifted particle when both dependency and complexity were low. When dependency and complexity are low, as in the sentence, 'the boy will finish his meal up,' processing is facilitated by shifting the particle, since the NP 'his meal' is encountered sooner than in the 
case of the adjacent particle 'the boy will finish up his meal.' Sentences such as 'the boy will finish his meal up,' where the particle is shifted, maximize the syntactic domain, at the expense of the semantic domain for 'finish' and 'up.' However, since the cost of moving the particle is not high, given the low dependency relationship between 'finish' and 'up', as well as the low complexity of the NP 'his meal,' the processing preference is for the shifted construction with the particle separated from the verb. However, if the dependency between the verb and particle were high, as in 'chew out,' or the NP were complex, as in a genitive relative clause, or both things were true, the preference would be very strong for keeping the verb and particle adjacent.

These results are most likely due to the differential weighting of factors when they are in opposition. When there is a high semantic dependency relationship between verb and particle, adjacency facilitates the semantic understanding of the entire verb phrase. Adjacency also assists processing when the direct object NP is complex since these NPs is difficult to process, especially when they intervene between highly dependent verbs and particles. However, when both dependency and complexity are low, processing is facilitated by minimization of the syntactic domain, with shifted sentences inducing a one word syntactic recognition benefit.

\subsection{Benefits of Adjacency}

When examining the interaction between adjacency and complexity, it is clear that adjacent structures facilitate processing since reading times are faster regardless of noun phrase complexity. In the shifted sentences, reading times slow as complexity increases, indicating that those relative clauses that minimize the distance between head and gap (i.e., subject relatives) are more easily processed than those that do not (i.e., direct object and genitive relatives). Listeners' sensitivity to the complexity of the direct object NP demonstrates that the grammaticalization of structures as defined by the complexity hierarchy of Keenan and Comrie (1977) affects processing. These results are also consistent with findings showing that processing is facilitated by shifting long or complex structures to the end of a sentence (Stallings et al, 1998).

When all three factors are examined together, there seems to be an overwhelming tendency to minimize the semantic dependency domain at the expense of the syntactic one, by keeping verb and particle adjacent. As dependency increases, adjacent particles facilitate processing and this effect is emphasized as complexity increases. If the particle is shifted, and dependency and complexity increase, the listener has to keep both the very dependent semantic relationship in memory, as well as the complex structure of the relative clause. Reading times slow dramatically since working memory load is very heavy (cf. Gibson, 2000). This exaggerated effect is shown in the word-by-word analysis in Figure 8. Taken together, these findings indicate that different factors interact to facilitate processing, resulting in 
the minimization of the domain with the most processing benefit.

The results also suggest that the plausibility of the direct object as the head noun plays an important role when semantically related structures are shifted apart. As mentioned earlier, shifting the particle in dependent structures with plausible head nouns could result in ambiguity and misinterpretation of the direct object noun phrase, leading to the need for reanalysis at the shifted particle. According to Lewis and Nakayama (2002), the speed up found for the particle when the head noun was implausible provides evidence that expectation does play a role in processing efficiency. The parse of an implausible head noun results in an expectation of the particle, thus when the particle is encountered and matches the expectation, processing is facilitated. Thus, hearing the sentence 'The teacher will chew the students out' leads to a slow-down at 'the students' initially, followed by a speed-up when the particle 'out' is encountered.

Results from the plausibility analysis also reinforced the notion that processing of sentences is greatly facilitated by the complete interpretation of verb and particle when they are adjacent. Therefore, it is understandable that the semantic relationships tend to thwart the inclination to minimize syntactic domains at the sentence level, especially when that minimization only results in a one word difference in recognition time. This notion is supported by the pervasive tendency, found throughout this study, to process adjacent sentences more easily.

These findings clearly demonstrate the correlation between processing ease and domain minimization, especially that of the semantic domain and the distance between head and gap in relative clauses.

\subsection{Benefits of Particle Shifting}

Based on the findings just discussed, it seems unclear as to why anyone would ever prefer to have a particle shifted to the end of a sentence. However, in English, we have not yet grammaticalized particle adjacent structures, and permit the option to shift a particle away from its verb. In order to understand the benefits of shifting the particle, it is important to stress that many factors, other than the three explored in this paper, influence verb-particle placement. For example, researchers have described several phonological, syntactic, semantic, pragmatic, and discourse factors that affect particle placement (e.g., Bolinger, 1971; Gries, 1999, 2002; Live, 1965; van Dongern, 1919).

More specifically, Gries (1999) has suggested that the particle in the shifted position is preferred when the sentence includes a pronoun as the direct object (e.g., 'the teacher will pass it out' versus 'the teacher will pass out it’*). Also, particle position may be affected by discourse factors, such that the particle is preferred in the shifted position when the direct object contains new or important information and thus, must come earlier in a sentence. Stress or intonation can also play a role in particle shifted, for example, if stressing the completive action in the sentence 'finish your meal up.' 
I suggest that it is important to examine other performance factors that affect sentence processing. Our study only looked at the interaction of three factors. Extensive research needs to be conducted to understand the effect and interaction of many of the factors not explored in this paper. Of particular interest is the effect of discourse factors (e.g., previous context) on comprehension of dependent, yet shifted verb-particle constructions.

The results from our study indicate that the number and kind of factors involved in any one sentence can influence the comprehension of sentences in many ways. I also suggest that the effect of any factor on processing will vary not only by other factors, but also by construction used. In different constructions, the benefit of adjacency may not be as high since verb-particle constructions rely heavily on one word order for proper interpretation. Dative constructions, for example, are easily interpreted in either order (e.g., 'the boy will throw the pretty girl the orange ball' versus 'the boy will throw the orange ball to the pretty girl') and do not have such a strong semantic dependency relationship between phrases.

Therefore, although it was not clear from the results of this study (which included two factors, namely direct object NP complexity and verb type (dependent versus independent), which strongly influence efficiency of the adjacent particle), there are several factors which can facilitate processing for the shifted particle and different constructions which can affect adjacency preferences.

\subsection{Processing Efficiency and Grammaticalization}

Results from this research show that less complex sentences (i.e., adjacent, low-dependency verb-particle constructions with direct object NP's of low complexity) do indeed maximize processing efficiency, as suggested by Hawkins (2004). These results support the idea that those structures which are more common across languages of the world (subject relatives and adjacent dependent constituents) are those that are more easily processed by individuals (c.f. Keenan \& Hawkins, 1987). More specifically, the findings from the experiments presented here suggest that when a language allows for ordering options, as in verb-particle constructions in English, processing is affected by different syntactic and semantic relationships within and between the various sentence constituents. Therefore, preferred structures can compete against one another when many domains interact in one sentence. This interaction of syntactic and semantic domains may provide insight into why certain structures are grammaticalized, while others are not.

Finally, I would like to emphasize the fact that this account of language grammaticalization and the relationship to individual processing ease is a probabilistic one. That is, the tendency across languages should be to fix as grammatical those structures that are easier to process, however, certain difficult constructions may remain if other aspects of the language push the system to retain a structure. Individual structures, such as English verb-particle constructions, must be considered in the context of the whole 
language.

\section{Appendix A}

Sample set of mean similarity ratings for verb/verb particle pairs

\begin{tabular}{ccc}
\hline Verb & Verb Particle & Mean Similarity Rating (SD) \\
\hline Start & start up & $8.50 \quad(0.50)$ \\
Count & count off & $6.75 \quad(1.88)$ \\
Block & block out & $5.75(2.62)$ \\
Smooth & smooth over & $4.64 \quad(2.30)$ \\
Shoot & shoot up & $3.48 \quad(2.04)$ \\
Throw & throw up & $2.52 \quad(1.73)$ \\
\hline
\end{tabular}

Note: 1 = very dissimilar, 9 = very similar.

\section{Appendix B}

Mean response latencies for target words by prime types and degree of prime-target similarity

\begin{tabular}{cccc}
\hline & \multicolumn{3}{c}{ Prime-Target Similarity } \\
\cline { 2 - 4 } Prime Type & Low & Mid & High \\
\hline Unrelated control (cast off/throw) & 550 & 553 & 557 \\
Related test (throw up/throw) & 543 & 532 & 537 \\
Unrelated-Related & $\mathbf{7}$ & $\mathbf{2 1}^{*}$ & $\mathbf{2 0}$ \\
\hline
\end{tabular}

\section{References}

Bolinger, D. The Phrasal Verb in English. Cambridge, MA: Harvard University Press; 1971.

Comrie, B. Language Universals and Linguistic Typology. Chicago, IL: University of Chicago Press; 1981.

Dehé, N., Jackendoff, R., McIntryre, A., Urban, S. Verb-Particle Explorations. Berlin: de Gruyter; 2002.

Gibson, E. Linguistic complexity: Locality of syntactic dependencies. Cognition,1998; 68:1-76.

Gibson, E. The dependency locality theory: A distance-based theory of linguistic complexity. In Y. Miyashita; A. Marantz (Eds.), Image, Language, Brain. Cambridge, MA: MIT Press. 2000 p. 95-126.

Gonnerman, L.M.; Hayes, C.R. The professor chewed the students... out: Effects of dependency, length, and adjacency on word order preferences in sentences with verb particle constructions. In Proceedings of 
the Twenty-Seventh Annual Conference of the Cognitive Science Society. Mahwah, NJ: Erlbaum; 2005. 21-23 July. p. 785-790.

Gries, S. Particle movement: A cognitive and functional approach. Cognitive Linguistics. 1999; 10:105145.

Gries, S. The influence of processing on syntactic variation: Particle placement in English. In: Dehe, N., Lackendoff, R., McIntyre, A., \& Urban, S. (eds.), Verb-Particle Explorations. Berlin: Mouton de Gruyter; 2002. p. 269-288

Hawkins, J.A. A Performance Theory of Order and Constituency. Cambridge: Cambridge University Press.1994.

Hawkins, J.A. Why are categories adjacent? Journal of Linguistics. 2001; 37:1-34.

Hawkins, J.A. Efficiency and Complexity in Grammars. Oxford: Oxford University Press. 2004.

Keenan, E. L. \& Comrie, B. Noun phrase accessibility and universal grammar. Linguistic Inquiry. 1977; 8:63-99.

Keenan, E. L. \& Hawkins, S. The psychological validity of the accessibility hierarchy. In E. Keenan (Ed.), Universal Grammar: 15 Essays. London: Croom Helm; 1987. p.60-85.

Lewis, R. \& Nakayama, M. Syntactic and positional similarity effects in the processing of Japanese embeddings. In: M. Nakayama (Ed.) Sentence Processing in East Asian Languages. Stanford: CSLI Publicaitons; 2002. p.85-111.

Live, A. The discontinuous verb in English. Word. 1965; 21:428-451.

Lohse, B., Hawkins, J.A., \& Wasow, T. Domain minimization in English verb-particle constructions. Language. 2004; 80(2):238-261.

Stallings, L.M., MacDonald, M.C., \& O’Seaghdha, P.G. Phrasal ordering constraints in sentence production: Phrase length and verb disposition in heavy-NP shift. Journal of Memory and Language. 1998; 39:392-417.

Van Dongen, W. A. Sr. He puts on his hat and he puts his hat on. Neophilologus.1919; 4,:322-353.

Wasow, T. Remarks on grammatical weight. Language Variation and Change.1997; 9:81-105.

Yamashita, H. \& Chang, F. “Long before short” preference in the production of a head final language. Cognition.2001:81(2), B45-B55. 